\title{
The Superhot, Superdense, Supersymmetric Universe ${ }^{a}$
}

\author{
A. RIOTTO \\ NASA/Fermilab Astrophysics Center, \\ Fermilab National Accelerator Laboratory, Batavia, Illinois 60510-0500, USA
}

\begin{abstract}
In this talk the following question is addressed: are internal symmetries necessarily restored at high temperature in supersymmetric theories? Contrary to the general belief, we argue that the answer is no when systems possess a net background charge. Our findings are exemplified on abelian models, for both cases of global and local symmetries and possible cosmological implications are also discussed.
\end{abstract}

\section{THE PREHISTORIC ERA}

When heated up, physical systems undergo phase transitions from ordered to less ordered phases. This deep belief, encouraged by everyday life experiences, would tell us that at high temperature spontaneously broken symmetries of high energy physics get restored. This, in fact, is what happens in the Standard Model (SM) of electroweak interactions. Whether or not true in general is an important question in its own right, but it also has a potentially dramatic impact on cosmology. Namely, most of the extensions of SM tend to suggest the existence of the so called topological defects and it is known that two types of such defects, i.e.domain walls and monopoles pose cosmological catastrophe. More precisely, they are supposed to be produced during phase transitions at high temperature $T \mathrm{t}$ and they simply carry too much energy density to be in accord with the standard big-bang cosmology. One possible way out of this problem could be provided by eliminatigg phase transitions if possible. In fact, it has been known for a long time 1 that in theories with a more than one Higgs field (and the existence of topological defects requires more than one such field in realistic theories) symmetries may remain broken at high $T$, and even unbroken ones may get broken as the temperature is increased. This offers a simple way out of the domain wall problem $\mathrm{B}$, whereas the situation regarding the monopole problem is somewhat less clear 1 .

Unfortunately, the same mechanism seems to be inoperative in supersymmetric theories. Whereas supersymmetry (SUSY) itself gets broken at high $T$, internal symmetries on the other hand get nefessarily restored. This has been proven at the level of renormalizable theories 5 . The argument goes as follows: in supersymmetric theories at finite temperature, the leading term in the effective potential is given by $T^{2}\left(\operatorname{Tr} \mathcal{M}_{f}^{\dagger} \mathcal{M}_{f}+\operatorname{Str} \mathcal{M}^{2}\right)$, where $\mathcal{M}_{f}$ is the mass

${ }^{a}$ Talk given at the SUSY97 Conference, May 27-31, Philadelphia PA, USA. 
matrix of the femionic degrees of freedom and $\operatorname{Str} \mathcal{M}^{2}$ is a field-independent quantity proportional to the soft breaking mass terms. It is easy to show that $\delta \operatorname{Tr} \mathcal{M}_{F} \mathcal{M}_{f}^{\dagger} / \delta \varphi_{i}=0$ gives $\left\langle\varphi_{i}\right\rangle=0$ 国.

Recent attempt to evade this argument using higher dimensional nonrenormalizable operators 6 , has been shown not to work 6 .

\section{THE DARK AGES}

About a year ago, at the SUSY96 Conference, all these considerations led my friends Borut Bajc and G. Senjanović to whisper (or, in other words, with the same tone as when you say Adelante Pedro, ma con judicio, A. Manzoni, I promessi sposi) that "... internal symmetries in supersymmetric theories seem to get restored at high temperature, even in the presence of non-renormalizable

interactions. However, one must admit that the proof offered is valid only for a single chiral superfield. We suspect that the above is true in general....".

As we know, dark ages are necessarily followed by a delightful period of renaissance. Luckily enough, that suspect has been shown recently to be false.

\section{THE RENAISSANCE ERA}

All the considerations mentioned above about SUSY and broken symmetries at high temperatures have an important assumption in common: the chemical potential is taken to be zero. In other words, it is assumed the vanishing of any conserved charge. What is the fate of internal symmetries at high temperatures in SUSY theories if we relax the assumption of vanishing conserved charge in the system? The answer to this question has been recently given in 6 . Actually, the answer was already known in nonsupersymmetric theories where it has been proven that the background charge asymmetry may postpone symmetry restoration at high temperature 10 , and even more remarkably that it can lead to symmetry breaking of internal symmetries, both in cases of global 11 and local symmetries 12 at arbitrarily high temperatures. This is simply a consequence of the fact that, if the conserved charge stored in the system is larger than a critical value, the charge cannot entirely reside in the thermal excited modes, but it must flow into the vacuum. This is an indication that the expectation value of the charged field is non-zero, i.e. that the symmetry is spontaneously broken. Furthermore, from the work of Affleck and Dine 13 we know that there is nothing unnatural about large densities in SUSY theories.

To leave the medieval age and come up to the air, let us warm up with the simplest supersymmetric model with a global $U(1)$ symmetry is provided by a 
chiral superfield $\Phi$ and a superpotential

$$
W=\frac{\lambda}{3} \Phi^{3} .
$$

It has a global $U(1) R$-symmetry under which fields transform as

$$
\phi \rightarrow \mathrm{e}^{i \alpha} \phi \text { and } \psi \rightarrow \mathrm{e}^{-i \alpha / 2} \psi,
$$

where $\phi$ and $\psi$ are the scalar and the fermionic component of the superfield $\Phi$. Thus the fermionic and bosonic charges are related by $2 Q_{\psi}+Q_{\phi}=0$, or in other words the chemical potentials satisfy the relation

$$
\mu \equiv \mu_{\phi}=-2 \mu_{\psi} .
$$

The presence of a nonvanishing net $R$-charge leads to a mass term $-\mu^{2} \phi^{\dagger} \phi$ for the scalar field with a "wrong" (negative) sign after canonical momenta relative to the boson degrees of freedom have been integrated out in the path integralle. The crucial new ingredient here is that such a bosonic contribution to the mass squared term already appears at the tree-level (and therefore is not depending upon $\lambda$ ), while the $\mu^{2}$ dependent part of the fermionic contribution to the mass squared term only appears at the one-loop level and is therefore suppressed by $\lambda^{2}$. To see this explicitly, one has to compute the fermionic-loop tadpole at finite temperature and chemical potential. Performing the summation over the Matsubara modes and integrating over the momentum $p$ one may show that for a small Yukawa coupling $\lambda \ll 1$ and $\mu<T$, the fermionic contribution in the chemical potential term to the mass squared is of order of $\lambda^{2} \mu^{2} \phi^{\dagger} \phi$ and therefore suppressed for small $\lambda$. We refer the reader to ref. 14 for more details. As a result, the fermionic degrees of freedom cannot compensate the genuine term $-\mu^{2} \phi^{\dagger} \phi$ originated by the bosonic partners. At finite temperature and finite chemical potential SUSY is broken.

The one-loop high temperature for small Yukawa coupling $\lambda \ll 1$ reads (for $\mu<T$ )

$$
V_{T}(\phi)=\left(-\mu^{2}+\frac{1}{2} \lambda^{2} T^{2}\right) \phi^{\dagger} \phi+\lambda^{2}\left(\phi^{\dagger} \phi\right)^{2} .
$$

Obviously, for $\mu^{2}>\lambda^{2} T^{2} / 2$ the symmetry is spontaneously broken at high temperature and the field $\phi$ gets a vacuum expectation value (VEV)

$$
\langle\phi\rangle^{2}=\frac{\mu^{2}-\frac{\lambda^{2}}{2} T^{2}}{\lambda^{2}} .
$$

This result is valid as long as the chemical potential $\mu$ is smaller than the scalar mass in the $\langle\phi\rangle$-background, i.e. $\mu^{2}<m_{\phi}^{2}=2 \lambda^{2}\langle\phi\rangle^{2}$ 11. This in turn implies 
$\mu>\lambda T$. In short, for a perfectly reasonable range

$$
\lambda T<\mu<T
$$

the original $U(1)_{R}$ global symmetry is spontaneously broken and this is valid at arbitrarily high temperatures (as long as the approximation of $\lambda$ small holds true).

Notice that, in all the above we have assumed unbroken supersymmetry. When supersymmetry is softly broken, $U(1)_{R}$ gets also explicitly broken because of the presence of soft trilinear scalar couplings in the Lagrangian. Therefore, the associated net charge vanishes and the reader might be worried about the validity of our result. However, the typical rate for $U(1)_{R}$-symmetry breaking effects is given by $\Gamma \sim \widetilde{m}^{2} / T$, where we have indicated by $\widetilde{m} \sim 10^{2}$ $\mathrm{GeV}$ the typical soft SUSY breaking mass term. Since the expansion rate of the Universe is given by $H \sim 30 T^{2} / M_{P \ell}, M_{P \ell}$ being the Planck mass, one finds that $U(1)_{R}$-symmetry breaking effects are in equilibrium and the net charge must vanish only at temperatures smaller than $T_{\mathrm{SS}} \sim \widetilde{m}^{2 / 3} M_{P \ell}^{1 / 3} \sim 10^{7} \mathrm{GeV}$. Therefore, it is perfectly legitimate to consider the presence of a nonvanishing $R$-charge at very high temperatures even in the case of softly broken SUSY.

Thus, we have provided a simple and natural counterexample to the theorem of the restoration of internal symmetries in supersymmetry $\mathrm{g}$. This is a consequence of the fact that the charge cannot be stored in the thermal excited modes, but it must reside in the vacuum and this is an indication that the expectation value of the charged field is non-zero, i.e. that the symmetry is spontaneously broken.

What about gauge symmetries? It has been known for a long time 12 that a background charge asymmetry tends to increase symmetry breaking in the case of a local gauge symmetry in nonsupersymmetric theories. In his work, Linde has shown how a large fermion number density would prevent symmetry restoration at high temperature in both abelian 12 and nonabelian theories 15. The essential point is that the external charge leads to the condensation of the gauge field which in turn implies the nonvanishing VEV of the Higgs field. This phenomenon may be easily understood if one recalls that an increase of an external fermion current $\mathbf{j}$ leads to symmetry restoration in the superconductivity theory 16 . In gauge theories symmetry breaking is necessarily a function of $j^{2}=j_{0}^{2}-\mathbf{j}^{2}$, where $j_{0}$ is the charge density of fermions. An increase of $j_{0}$ is therefore accompanied by an increase of symmetry breaking 12. We now demonstrate that this phenomenon persists in supersymmetric theories, at least in the case of abelian symmetry.

The simplest model is based on $U(1)$ supersymmetric local gauge symmetry. The minimal anomaly free matter content consists of two chiral superfields 
$\Phi^{+}$and $\Phi^{-}$with opposite gauge charges and the most general renormalizable superpotential takes the form

$$
W=m \Phi^{+} \Phi^{-} .
$$

Notice that the symmetry is not spontaneously broken at zero temperature. Since there is no Yukawa interaction, there is also a global $U(1) R$-symmetry, under which the bosons have, say, the same charge and fermions are invariant. Furthermore at very high temperature, $T>m^{2 / 3} M_{P \ell}^{1 / 3}$, the fermion mass can be neglected and we get also a chiral $U(1)$ symmetry under which the bosons are invariant.

We may now suppose for simplicity that there is a net background charge density $j_{0}$, with the zero current density and that it lies entirely in the fermionic sector. In other words we assume the background charge to be in the form of the chiral fermionic charge. Thus only the fermions have a nonvanishing chemical potential.

Equally important, we assume that the gauge charge of the Universe is zero, just as in 12 . In the realistic version of this example, one would imagine the gauge charge to be the electromagnetic one and the chiral fermionic charge to be, say, the lepton charge in the MSSM. We know from observation that the electromagnetic charge of the Universe vanishes to a good precision. Thus we have to minimize the action with the constraint that the electric field is zero. What will happen is that some amount of bosonic charge will get stored into the vacuum in order to compensate for the fermionic one and achieve the vanishing of the electric field. In this way, the total $U(1)$ charge density of the system including the charge of the condensate is equal to zero even if symmetry is broken and the gauge forces are short-range ones. This is the principal reason behind the resulting spontaneous symmetry breaking of the local gauge symmetry, as we show below. It is crucial thus to have some nonvanishing external background charge, i.e. the model should have some extra global symmetry as provided by our chiral symmetry.

We can obviously take $A_{i}=0$ in the vacuum and treat $A_{0}$ on the same footing with the scalar fields $\phi^{ \pm}$(due to the net charge $A_{0}$ cannot vanish in the vacuum). If we now integrate out $A_{0}$ using its equation of motion, assuming the electric field to be zero, we can then compute the high temperature potential for the scalar fields in question at high temperature and large charge density with the following result

$$
\begin{aligned}
V_{\mathrm{eff}}(T) & =\frac{g^{2}}{2} T^{2}\left(\left|\phi^{+}\right|^{2}+\left|\phi^{-}\right|^{2}\right) \\
& +\frac{g^{2}}{2}\left(\left|\phi^{+}\right|^{2}-\left|\phi^{-}\right|^{2}\right)^{2}
\end{aligned}
$$




$$
+\frac{1}{2} \frac{j_{0}^{2}}{2\left(\left|\phi^{+}\right|^{2}+\left|\phi^{-}\right|^{2}\right)+T^{2}}
$$

where we have taken $T \gg m$ and we have included both scalar and fermionic loop contributions in the $T^{2}$ mass term for $A_{0}$. Now, except for the $D$-term, the rest of the potential depends only on the sum $\phi^{2} \equiv\left|\phi^{+}\right|^{2}+\left|\phi^{-}\right|^{2}$, and thus the energy is minimized for the vanishing of the $D$-term potential, i.e. for $\left|\phi^{+}\right|^{2}=\left|\phi^{-}\right|^{2}$. It is easy to see that in this case the effective potential has two extrema:

$$
\phi=0 \quad \text { and } \quad \phi^{2}=\frac{j_{0}}{\sqrt{2} g T}-\frac{T^{2}}{2} .
$$

The second extremum obviously exists only for

$$
j_{0}>\frac{g T^{3}}{\sqrt{2}} .
$$

Moreover in that case it is an absolute minimum, while $\phi=0$ is a maximum. Now, we can rephrase the above condition in the language of the chemical potential (using $g_{*}=4$ )

$$
\mu>g T .
$$

For $g \ll 1$, which is of our interest, $\mu$ easily satisfies the condition $\mu<T$. As noted above, since Yukawa interactions are absent, the role of the external charge may have been played by the $R$-charge in the scalar sector, the two scalars being equally charged under this symmetry. In such a case, the analysis requires careful handling because of issues related to gauge invariance. In a future publication 17 we will extensively explore this issue as weel generalize our results to theories containing an arbitrary number of abelian symmetries and to nonabelian theories.

\section{THE FUTURE DAYS}

We hope to have convinced the audience that internal symmetries in supersymmetric theories, contrary to the general belief, may be broken at high temperature, as long as the system has a nonvanishing background charge. The examples we have provided here, based on both global and local abelian symmetries, are natural and simple and should be viewed as prototypes of more realistic theories. The necessary requirement for the phenomenon to take place is that the chemical potential be bigger than a fraction of temperature on the order of (1-10)\%. Notice that this is by no means unnatural. In the expanding universe the chemical potential is proportional to temperature, and thus unless 
zero for some reason, $\mu / T$ is naturally expected to be of order one. More important, this chemical potential could be zero today, all that is needed is that it is nonvanishing at high temperature. We have seen how soft supersymmetry breaking may naturally provide such a scenario if there is some nonvanishing external charge.

Now, it is well known that in suspersymmetry the existence of flat directions may lead to large baryon and lepton number densities at very high temperature 13 . The most natural candidate for the large density of the universe is the lepton number that may reside in the form of neutrinos. It should be stressed that a large lepton number is perfectly consistent with the ideas of grand unification. It can be shown that in $S O(10)$ one can naturally arrive at a small baryon number and a large lepton number 19 .

In any case, we wish to be even more open minded and simply allow for a charge density without worrying about its origin. It is noteworthy that a large neutring number density may persist all the way through nucleosynthesis up to today 18. This has been used by Linde 15 in order to argue that even in SM the $S U(2)_{L} \otimes U(1)_{Y}$ symmetry may not be restored at high temperature. Since SUSY, as we have seen, does not spoil the possibility of large chemical potentials allowing symmetry breaking at high $T$, it is important to see if our results remain valid in the case of nonabelian global and local symmetries. This work requires particular attention due to the issues related to gauge invariance and is now in progress 17 .

We should stress that there is more than a sole academic interest to the issue discussed in this paper. If symmetries remain broken at high temperature, there may be no domain wall and monopole problems at all. Furthermore, it is well known that in SUSY grand unified theories symmetry restoration at high temperature prevents the system from leaving the false vacuum and finding itself in the broken phase at low $T$. This is a direct consequence of the vacuum degeneracy characteristic of supersymmetry which says that at zero temperature the SM vacuum and the unbroken GUT symmetry one have the same (zero) energy. If the symmetry is restored at high $T$, one would start with the unbroken symmetry in the early universe and would thus get caught in this state forever. Obviously, if our ideas hold true in realistic grand unified theories, this problem would not arise in the first place. Moreover, we know that the presence of anomalous baryon number violating precesses induced by sphaleron transitions pose a serious problem for the survival of the baryon asymmetry in the early Universe. However, sphalerons are active at high temperatures only if the standard model gauge symmetry is restored. The presence of a nonvanishing (lepton?) conserved charge in the minimal supersymmetric standard model might cause the symmetry nonrestoration of 
the gauge symmetry when the system is heated up, as orioinally suggested by Liu and Segrè for the nonsupersymmetric standard model20. This would allow to freeze the baryon number violating processes and to preserve the baryon asymmetry generated at the GUT scale.

\section{Acknowledgments}

It is with immense pleasure that we thank my collaborators Borut Bajc and Goran Senjanović with whom this journey out of the dark ages towards a so exciting period has begun. This work is supported by the DOE and NASA under Grant NAG5-2788.

1. T.W.Kibble, J.Phys., A9 (1976) 1987; Phys. Rep. 67 (1980) 183.

2. S.Weinberg, Phys. Rev., D9 (1974) 3357; R.N.Mohapatra and G.Senjanović, Phys. Rev. Lett. 42 (1979) 1651; Phys. Rev. $\mathbf{D 2 0}$ (1979) 3390.

3. G. Dvali and G. Senjanović, Phys. Rev. Lett. 74 (1995) 5178.

4. G. Dvali, A. Melfo and G. Senjanović, Phys. Rev. Lett. 75 (1995) 4559; G. Bimonte and L. Lozano, Phys.Lett. B366,248 (1996); Nucl.Phys. B460, 155 (1996).

5. H. Haber, Phys. Rev. $\mathbf{D 2 6}$ (1982) 1317 ; M. Mangano Phys.Lett. B147 (1984) 307

6. G. Dvali and K. Tamvakis, Phys. Lett. B378 (1996) 141.

7. B. Bajc, A. Melfo and G. Senjanović, Phys. Lett. $B 387$ (1996) 796, hep-ph/9607242.

8. B. Bajc and G. Senjanović, talk given by G. Senjanovic at SUSY96, University of Maryland, 1996; Nucl. Phys. Proc. Suppl. 52A (1997) 246.

9. A. Riotto and G. Senjanović, FERMILAB-PUB-97-020-A, hepph/9702319, to be published in Phys. Rev. lett.

10. H.E. Haber and H.A. Weldon, Phys. Rev. D25 (1982) 502.

11. K.M. Benson, J. Bernstein and S. Dodelson, Phys. Rev. D44 (1991) 2480 .

12. A.D. Linde, Phys. Rev. D14 (1976) 3345.

13. I. Affleck and M. Dine, Nucl. Phys. B249 (1985) 361.

14. B.J. Harrington and A. Yildiz, Phys. Rev. Lett. 33 (1974) 324.

15. A.D. Linde, Phys. Lett. B86 (1979) 39.

16. See for instance, P.G. De Gennes, Superconductivity of Metals and Alloys (Benjamin, New York, 1966).

17. B. Bajc, G. Senjanović and A. Riotto, in preparation. 
18. Large neutrino chemical potentials $|\mu|$ for the three neutrino species affects the energy density in the neutrino species, and thereby the expansion rate of the Universe, dangerously increasing the primordial production of ${ }^{4} \mathrm{He}$. However, it is possible to dial in the desired ${ }^{4} \mathrm{He}$ abundance by the appropriate sign of the neutrino chemical potential, see, for instance, E.W. Kolb and M.S. Turner, The Early Universe, AddisonWesley Ed. (1990), p. 107.

19. J. Harvey and E. Kolb, Phys. Rev. D24 (1981), 2090.

20. J. Liu and G. Segrè, Phys. Lett. B338 (1994), 259. 